\title{
Electromechanical Resonances of SiC and AlN Beams under Ambient Conditions
}

\author{
K. Brueckner ${ }^{1}$, Ch. Förster ${ }^{2}$, K. Tonisch ${ }^{2}$, V. Cimalla ${ }^{2}$, O. Ambacher ${ }^{2}$, R. Stephan ${ }^{1}$, K. Blau ${ }^{1}$, and \\ M. A. Hein ${ }^{1}$ \\ ${ }^{1}$ Department for RF and Microwave Techniques \\ ${ }^{2}$ Department of Nanotechnology \\ Centre for Micro- and Nanotechnologies \\ Technische Universität Ilmenau, P.O. Box 100565, 98684 Ilmenau, Phone +49 (3677) 69-1580
}

\begin{abstract}
MEMS resonators offer great potential for RF sensor and filter applications. A semiconductor process has been used to prepare $\mathrm{SiC}$ and $\mathrm{AIN}$ beam resonators. The metallised beams are excited by an RF current in a permanent magnetic field. The resonant response is detected by the induced voltage. Despite the weakness of the signal, accurate detection has been achieved in the time domain, under ambient conditions in a magnetic field of about $0.5 \mathrm{~T}$. The resonant response bears valuable information on the structural quality of the material and identifies potential for further improvement. The time domain technique presents an elegant approach to sensing applications.
\end{abstract}

\section{INTRODUCTION}

Semiconductor production processes originally designed for manufacturing integrated circuits can be used to make complex-shaped and well defined electromechanical structures. Even if the technology is based on planar concepts, the combination of etching and lift-off processes allows the design of various three-dimensional structures. These patterned semiconductors can form mechanical resonators and, by metallisation, be coupled to electrical circuits. The single-crystalline material allows for the preparation of surfaces and volumes with a low density of defects, which is essential for high mechanical quality factors. The mechanical oscillation of the resonators is affected by various physical parameters, e.g. mass, friction, tension, and temperature. Detecting these parameters electrically would make accessible a variety of sensor applications. With respect to a high sensitivity, in all cases, a high resonant frequency is beneficial. This requires small geometries which are conveniently provided by modern lithographic techniques. Among future applications of mechanical high-frequency resonators are ultrasensitive sensors for chemical or biological species $[1,2]$ but also filters and other RF applications $[3,4]$ that would benefit from the integration of active and passive components on the same chip.

\section{RESONATOR STRUCTURE}

Mechanical oscillations in semiconductor materials have been demonstrated by several groups, e.g., [5-7]. Typically, the mechanical resonance is stimulated by periodic energy supply, and the resulting vibration is detected electrically. In this paper, the basic resonator structure is a doubly clamped beam that is actuated mag-

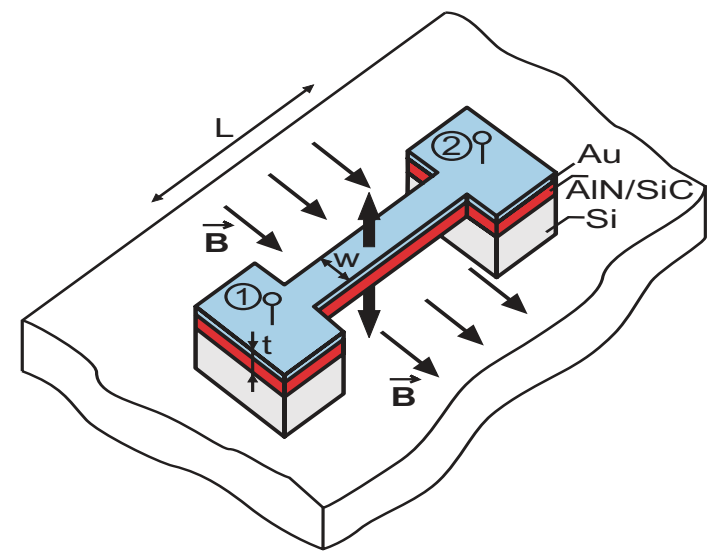

Fig. 1. Mechanical resonator of beam length $L$, width $w$ and thickness $t$, with contact areas 1 and 2, in an external magnetic field of flux density $B$. The bold arrows indicate the direction of the Lorentz-force and the resulting beam deflection.

netomotively. A Lorentz-force is generated by placing the beam in a permanent magnetic field and applying an RF current to it, as illustrated by Fig. 1 .

Using optical lithography, beams were prepared in various dimensions. They consist of a 50 to 200 -nm thick AlN layer, or a 200-nm thick SiC(100) layer, with a 50$\mathrm{nm}$ thick gold metallisation on top. The beams vary in length and width from 10 to $500 \mu \mathrm{m}$ and $500 \mathrm{~nm}$ to $8 \mu \mathrm{m}$, respectively. Based on the Euler-Bernoulli theory [5] and recent extensions [8], the fundamental resonant frequencies $f_{\text {res }}$ of the beams can be estimated by

$$
f_{\text {res }}=1.03 \sqrt{\frac{E}{\rho}} \cdot \frac{t}{L^{2}} \cdot c(\varepsilon),
$$

where $E$ is Young's modulus, $\rho$ the mass density, $t$ the thickness and $L$ the length of the beam. The factor $c(\varepsilon) \approx$ $\left[1+0.3 \times(L / t)^{2} \times \mathcal{E}\right]^{1 / 2}$ accounts for the effect of tensile or compressive axial strain $(\varepsilon>$ or $<0)$ on $f_{\text {res }}$ [8]. In addition to the analytical approach, the resonant frequencies were calculated numerically (for $\varepsilon=0$ ) using the commercial finite-element simulation software ANSYS $^{\circledR}$, leading to $f_{\text {res }}$-values ranging from $3 \mathrm{kHz}$ to $1 \mathrm{MHz}$ for $\mathrm{AlN}$, and $26 \mathrm{kHz}$ to $17 \mathrm{MHz}$ for $\mathrm{SiC}$.

\section{FABRICATION TECHNOLOGY}

Fig. 2 sketches the main steps of the MEMS fabrication. The 200-nm thick single-crystalline SiC films were 
grown by chemical vapour deposition under ultra-high vacuum $[9,10]$. A mixture of silane, ethene and hydrogen was used as chemical precursors. The $3 \mathrm{C}-\mathrm{SiC}(100)$ was grown heteroepitaxially on $\mathrm{Si}(100)$ at temperatures below $1000^{\circ} \mathrm{C}$. The reduction of the growth temperature lowers the residual strain of the $\mathrm{SiC}$ layers, a feature that is important for the processing and the electromechanical performance of the MEMS structures.

The AlN-films were DC-sputtered reactively on $\mathrm{Si}(111)$ substrates at $500^{\circ} \mathrm{C}$ in pure nitrogen atmosphere. Prior to the deposition, an ultrathin atomically flat $\mathrm{SiC}$ layer has been prepared by carbonisation by rapid thermal processing [11].

Electron-beam evaporation has been used to metallise both, the SiC and AlN coated wafers, with a $50 \mathrm{~nm} \mathrm{Au}$ layer (Fig. 2a). Afterwards, the resonator structures were patterned by a lift-off process using optical lithography (Fig. 2b).

The MEMS structures were dry etched (Fig. 2c and d) in an electron cyclotron resonance-stimulated plasma. The source operated at $2.45 \mathrm{GHz}$ with a magnetic flux density of $87.5 \mathrm{mT}$. Two independent gas-inlet-systems were used for the etch process. The first system enabled the plasma gases, $\mathrm{Ar}$ and $\mathrm{O}_{2}$, to enter the chamber near the plasma source. The second one controlled the flow of the fluorine gases required for the dry etching of the $\mathrm{SiC} / \mathrm{Si}$-structures [12], and of $\mathrm{C}_{2} \mathrm{H}_{4}$ and $\mathrm{H}_{2}$ for the nitride-based resonator beams, respectively.

a)

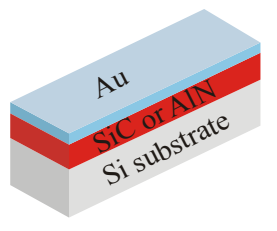

c)

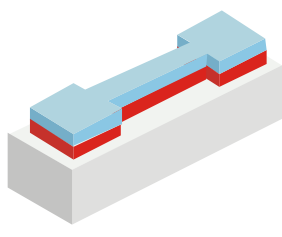

b)

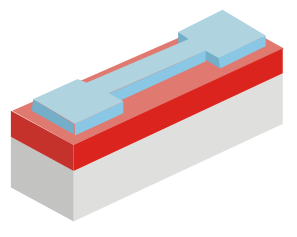

d)

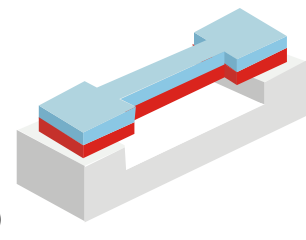

a)

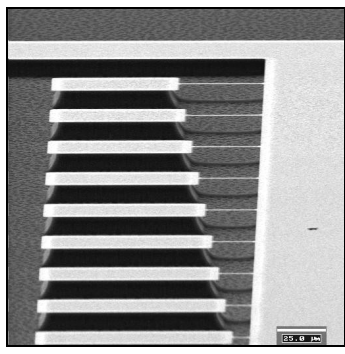

b)

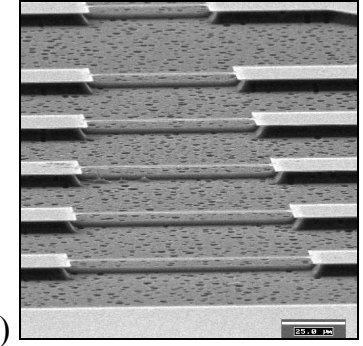

c)

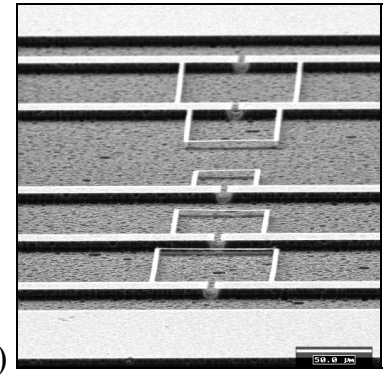

Fig. 3. Scanning-electron microscope (SEM) images of the doubly clamped resonators. (a) Set of 250-nm thick AlN-beams with lengths from 8 to $40 \mu \mathrm{m}$. (b) Set of 250-nm thick SiCbeams with lengths from 40 to $100 \mu \mathrm{m}$. (c) U-shaped SiC resonator structure with up to $160 \mu \mathrm{m}$ suspended cantilever length and $80 \mu \mathrm{m}$ transversal beam length.

SEM images of the achieved resonator structures are shown in Fig. 3a and $\mathrm{b}$ for $\mathrm{AlN}$ and $\mathrm{SiC}$, respectively. Freestanding U-shaped SiC-resonators are presented in Fig. 3c. Compared to doubly clamped beams of the same dimension, these structures have lower resonant frequencies, while the electrical output signal is higher because of the much higher mechanical vibration amplitudes. Using the U-shaped resonators, we initially verified the proof-of-principle as well as the mechanical stability of the structures [13]. In the following, we focus on the data obtained for the doubly clamped beams.

\section{MEASUREMENT SETUP}

As described in Sec. II, the oscillation of the beams is forced by an RF current at several $\mathrm{MHz}$, driven by a primary voltage of the order of Volts. The secondary voltage, induced by the periodic displacement, is much smaller than the primary voltage, of the order of $\mu \mathrm{V}$. Therefore, it is very difficult to detect as a tiny signal superimposed by the strong excitation signal. To overcome this problem, high magnetic flux densities of the order of several Teslas were used by some groups, or the resonators were cooled to cryogenic temperatures [5]. For two-port arrangements like the one sketched in Fig. 1, there will be always the problem to separate the secondary voltage from the excitation signal. Different approaches with balanced or bridge circuits have been reported recently [6].

We propose yet another solution to overcome this problem, namely by separating the excitation signal and the response of the resonator in the time domain. With a period of $T_{0}=1 \mathrm{~ms}$, the beam is excited by short voltage pulses of amplitude $U_{D}=300 \mathrm{mV}$, as sketched in Fig. 4a. The pulse duration $\Delta t$ is varied between 1 and $10 \mu \mathrm{s}$ in order to accommodate an integer multiple of resonant beam oscillations, hence maximising the output signal. 
Due to the short duration $\Delta t$ of the pulse, the thermal stress of the metallisation layer can be reduced significantly to about one percent, compared to a continuouswave excitation.

Fig. $4 \mathrm{~b}$ shows the equivalent circuit of our measurement setup. For small amplitudes, the motion of the beam can be described by a damped mass-spring system, which is represented in Fig. $4 \mathrm{~b}$ by its equivalent electrical analogue, a parallel resonant circuit (red coloured), where $C_{m}$ represents the resonator mass, $L_{m}$ its effective spring constant and $R_{m}$ the damping losses. $R_{e}$ is the ohmic resistance of the metallisation layer on top of the resonator beams. A rectifying diode was inserted, to decouple the generator with its $50 \Omega$ source impedance from the characteristic impedances of the resonators, which are of similar magnitude.

Fig. 5a shows a photograph of the assembly operated under ambient laboratory conditions. All resonator beams were contacted with coplanar RF probe tips. The magnetic field was provided by permanent magnets with a flux density of about $0.5 \mathrm{~T}$ at the location of the beam.

The free decay of the resonator can be monitored with an oscilloscope (Fig. 5b). The periodic response yields information on the resonant frequency $f_{\text {res }}$ and the loaded quality factor $Q$ of the resonator: For $T_{P}$ denoting the period of oscillation of the beam and $t_{1 / 2}$ the time for the decay of the induced voltage to one half of its initial value $U_{S}, Q$ is given by

$$
Q=\frac{\pi}{\ln 2} \cdot \frac{t_{1 / 2}}{T_{\mathrm{P}}} .
$$

Whereas the electrical resistance $R_{e}$ of the metallisation layer of the beams can be measured directly, the other parameters $C_{m}, L_{m}$ and $R_{m}$ can be extracted only by comparing the solution of the equation of motion of the system with the experimentally obtained decay curves. The
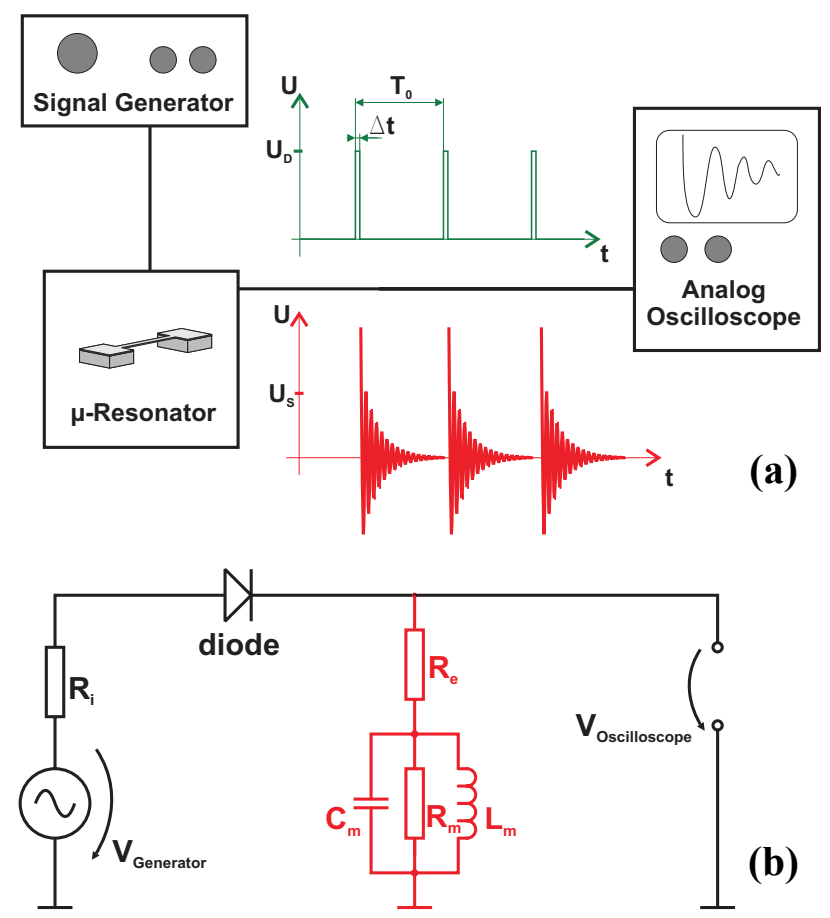

(b)

Fig. 4. (a) Schematic measurement setup, diagrams of excitation and read-out time signal. (b) Measurement circuit with resonator represented by its equivalent-circuit.
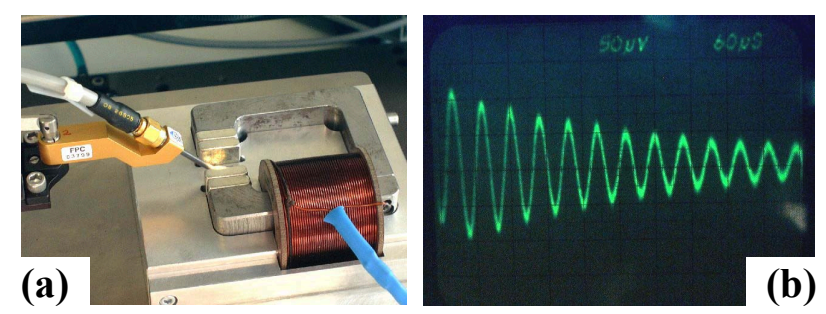

Fig. 5. (a) Photograph of a probed chip. (b) Typical oscilloscope trace of the response of a resonant beam to an excitation pulse, voltage scale: $50 \mu \mathrm{V} / \mathrm{div}$, time scale: $20 \mu \mathrm{s} / \mathrm{div}$.

parameters are necessary for computer simulation of the system. Typical values for the two materials used are shown in Table 1. The higher capacitance $C_{m}$ of the SiC resonator corresponds to the higher mass of the beam, the lower inductance $L_{m}$ to its higher spring constant. A higher resistance $R_{m}$ means less mechanical losses, but for Q-factor determination, $R_{m}$ has to be related to the resonant frequency of the beam.

\begin{tabular}{|c|c|c|}
\hline & $\begin{array}{c}\text { SiC beam } \\
(250-\mathrm{nm} \text { thick })\end{array}$ & $\begin{array}{c}\text { AlN beam } \\
(110-\mathrm{nm} \text { thick })\end{array}$ \\
\hline $\boldsymbol{f}_{\text {res }}(\mathrm{kHz})$ & $\mathbf{8 0 0}$ & $\mathbf{2 3 3}$ \\
\hline $\boldsymbol{Q}$ & 254 & 125 \\
\hline $\boldsymbol{R}_{\boldsymbol{e}}(\mathbf{\Omega})$ & 34.3 & 34.6 \\
\hline $\boldsymbol{R}_{\boldsymbol{m}}(\mathbf{m} \Omega)$ & 307 & 840 \\
\hline $\boldsymbol{L}_{\boldsymbol{m}}(\mathrm{nH})$ & $\mathbf{0 . 2 4}$ & 4.59 \\
\hline $\boldsymbol{C}_{\boldsymbol{m}}(\boldsymbol{\mu} \mathbf{F})$ & 164 & 102 \\
\hline
\end{tabular}

Tab. 1. Equivalent electrical circuit parameters for two selected resonator beams of identical length $L=125 \mu \mathrm{m}$ but different material. Blue: measured values, red: derived values.

\section{RESONANT FREQUENCIES AND QUALITY FACTORS}

The maximum induced voltages reached $120 \mu \mathrm{V}$ and were obtained for the longest beams, while the response of short beams dropped to as little as $10 \mu \mathrm{V}$ due to the smaller amplitudes of the mechanical oscillation. The measured $f_{\text {res }}$-values covered the range from 6 to $750 \mathrm{kHz}$ for the AlN beams, and 0.32 to $1.5 \mathrm{MHz}$ for the $\mathrm{SiC}$ beams. Fig. 6a compares the dependence of the resonant frequencies (left-hand scale), and the ratio of measured to calculated resonant frequencies, $v=f_{\text {res }} / f_{\text {sim }}$ (right-hand scale), on beam length for both materials. The resonant frequencies of the $\mathrm{SiC}$ beams are higher than for $\mathrm{AlN}$ beams of identical length, mainly because of the higher beam thickness and the higher Young's modulus. However, the piezoelectric properties of AlN bear the potential for an all-electrical actuation and detection [14], offering elegant solutions for miniaturised sensor applications without the need for magnetic fields.

We note a linear increase of the $v(L)$-curves. This implies that the resonant frequencies scale like $1 / L$ rather than $1 / L^{2}$ as expected for relaxed beams. According to Eq. (1), this behaviour is reminiscent of strained material. Similar conclusions were drawn for resonators made of polycrystalline $\mathrm{Si}$ [8]. For a given film thickness, the slope $\mathrm{d} v / \mathrm{d} L$ is a direct measure of the residual strain $\varepsilon$. Obviously, the AlN beams are much less strained $(\varepsilon \approx$ $\left.+5 \times 10^{-5}\right)$ than the SiC beams $\left(\varepsilon \approx+5 \times 10^{-4}\right)$. This difference could be caused by a higher density of defects in the 

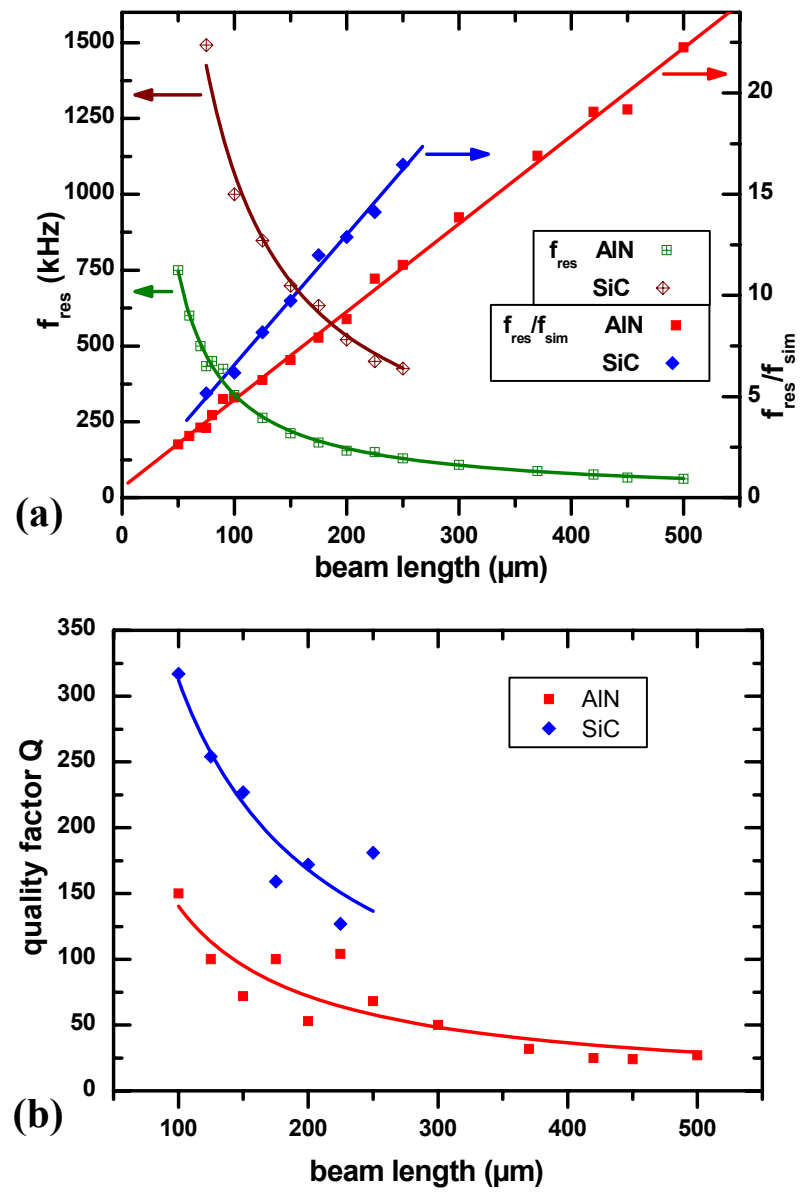

Fig. 6. (a) Frequency versus beam length for the doubly clamped $\mathrm{SiC}$ and $\mathrm{AlN}$ beams: Measured values and reciprocal curve fitting for $f_{\text {res }}(L)$ (left-hand scale), values and linear fit for $f_{\text {res }} / f_{\text {sim }}(L)$ (right-hand scale). (b) Measured quality factors versus beam length and reciprocal curve fitting for various $\mathrm{SiC}$ and AlN resonators.

sputtered AlN films compared to the evaporated SiC films, facilitating relaxation of residual strain.

The Q-factors derived from the data are displayed in Fig. 6b; the values around 200 compare favourably with the results of other groups operating resonators under ambient conditions [7]. Frictional damping by air is the most dominant loss mechanism, leading to decreasing Qvalues for increasing beam length. The SiC beams display clearly higher Q-values than the AlN resonators, which can be associated with by the differences of their material (Young's modulus E) and geometrical (beam thickness $t$ ) properties $[15,16]$.

\section{CONCLUSION}

We have successfully realised free-standing resonator beams of widely varied geometries from heteroepitaxial $\mathrm{SiC}$ and $\mathrm{AlN}$ films on Si. The resonant properties of the beams actuated under ambient conditions in a static magnetic field were investigated by a pulsed measurement scheme. By this technique, the weak resonant response could be separated from the strong excitation with high accuracy. The quality factors of the resonators, covering frequencies from 50 to $1500 \mathrm{kHz}$, reached values around 250. The moderate Q-values as well as linear scaling of the resonant frequencies with beam length indicate resid- ual tensile strain and hence the potential for further improvement. The superior elastic and piezoelectric properties of AlN compared to $\mathrm{SiC}$ favour, in principle, its application for integrated RF MEMS.

\section{ACKNOWLEDGEMENT}

This work has been funded by the German Science Foundation (DFG), Priority Programme 1157 (AM 105/2-1).

\section{REFERENCES}

[1] B. Ilic, H. G. Craighead, S. Krylov, W. Senaratne, C. Ober, and P. Neuzil, "Attogram detection using nanoelectromechanical oscillators," J. of Appl. Phys., vol. 95, no. 7, pp. 3694-3703, 2004.

[2] K. L. Ekinci, X. M. H. Huang, and M. L. Roukes, "Ultrasensitive nanoelectromechanical mass detection," Appl. Phys. Lett., vol. 84, no. 22, pp. 4469-4471, 2004.

[3] C. T.-C. Nguyen, "Vibrating RF MEMS for low power wireless communications," Proc. 2000 Int. MEMS Workshop (iMEMS'01), Singapore, pp. 21-34, 2001.

[4] S. Pourkamali, R. Abdolvand, and F. Ayazi, "A $600 \mathrm{kHz}$ electrically-coupled MEMS bandpass filter," IEEE MEMS'03, Kyoto, Japan, pp. 702-705, 2003.

[5] A. N. Cleland, M. Pophoristic, and I. Ferguson, "Singlecrystal aluminum nitride nanomechanical resonators," Appl. Phys. Lett., vol. 79, no. 13, pp. 2070-2072, 2001.

[6] K. L. Ekinci, Y. T. Yang, X. M. H. Huang, and M. L. Roukes, "Balanced electronic detection of displacement in nanoelectromechanical systems," Appl. Phys. Lett., vol. 81, no. 12, pp. 2253-2255, 2002.

[7] L. Sekaric, M. Zalalutdinov, R. B. Bhiladvala, A. T. Zehnder, J. M. Parpia, and H. G. Craighead, "Operation of nanomechanical resonant structures in air," Appl. Phys. Lett., vol. 81, no. 14, pp. 2641-2643, 2002.

[8] T. Ikehara, R. A. F. Zwijze, and K. Ikeda, "New method for an accurate determination of residual strain in polycrystalline silicon films by analysing resonant frequencies of micromachined beams," J. Micromech. Microeng., vol. 11, no. 1, pp. 55-60, 2001.

[9] R. S. Kern and R. F. Davis, "Deposition and doping of silicon carbide by gas-source molecular beam epitaxy," Appl. Phys. Lett., vol. 71, no. 10, pp. 1356-1358, 1997.

[10] Ch. Förster, V. Cimalla, O. Ambacher, and J. Pezoldt, "Low temperature chemical vapor deposition of $3 \mathrm{C}-\mathrm{SiC}$ on Si substrates," Mater. Sci. Forum, SiC Rel. Mater. 2004 (ECSCRM 2004), vol. 483-485, pp. 201-204, 2005.

[11] V. Cimalla, J. K. Karagodina, J. Pezoldt and G. Eichhorn, "Growth of thin $\beta$-SiC layers by carbonisation of Si surfaces by rapid thermal processing," Mater. Sci. Eng. B, vol. 29, pp. 170-175, 1994.

[12] P. H. Yih, V. Saxena, and A. J. Steckl, "A review of SiC reactive ion etching in fluorinated plasmas," phys. stat. sol. (b), vol. 202, pp. 605-642, 1997.

[13] K. Brueckner, R. Stephan, M. A. Hein, Ch. Foerster, V. Cimalla, and O. Ambacher, "Micro-electromechanical RF resonators from $\mathrm{SiC} / \mathrm{Si}$ heterostructures," MEMSWAVE 2004, Uppsala, Sweden, 30 June - 2 July 2004.

[14] B. Piekarski, D. DeVoe, M. Dubey, R. Kaul, and J. Con$\mathrm{rad}$, "Surface micromachined piezoelectric resonant beam filters," Sens. Actuators A, vol. 91, no. 3, pp. 313-320, 2001.

[15] H. Hosaka, K. Iato, and S. Kuroda, "Damping characteristics of beam-shaped micro-oscillators," Sens. Actuators A, vol. 49, no. 1-2, pp. 87-95, 1995.

[16] C. Zhang, G. Xu, and Q. Jiang, "Analysis of the air-damping effect on a micromachined beam resonator," Math. Mech. Solids, vol. 8, pp. 315-325, 2003. 